09,12

\title{
Резонансный перенос энергии в плотном массиве II-VI квантовых точек
}

\author{
(C) Т.В. Шубина, К.Г. Беляев, М.А. Семина, А.В. Родина, А.А. Головатенко, А.А. Торопов, С.В. Сорокин, \\ И.В. Седова, В.Ю. Давыдов, А.Н. Смирнов, П.С. Копьев, С.В. Иванов
}

Физико-технический институт им. А.Ф. Иофрфе РАН,

Санкт-Петербург, Россия

E-mail: shubina@beam.ioffe.ru

\begin{abstract}
Методами спектроскопии фотолюминесценции с временны́м и пространственным разрешением продемонстрирован ферстеровский резонансный перенос энергии в неоднородных плотных массивах эпитаксиальных квантовых точек $\mathrm{CdSe} / \mathrm{ZnSe}$, отличительной чертой которого является диполь-дипольное взаимодействие между основными экситонными уровнями маленьких квантовых точек и возбужденными уровнями больших точек, приводящее к эффективному сбору энергии и спектральной селекции ограниченного числа излучателей. Результаты теоретического моделирования оптических переходов в сфероидных квантовых точках с гауссовым потенциальным профилем согласуются с наблюдаемыми особенностями в оптических спектрах, вызванными изменением доминирующего механизма переноса энергии.
\end{abstract}

Работа выполнена при поддержке Российского научного фонда (проект № 14-22-00107).

\section{1. Введение}

Широкозонные квантовые точки (QDs) на основе соединений групп II-VI, создаваемые методом молекулярно-пучковой эпитаксии (MBE) посредством осаждения дробного количества монослоев (fractional monolayer, $\mathrm{ML}) \mathrm{CdSe}$ в широкозонную матрицу активно исследуются с конца прошлого века [1]. Планарные массивы $\mathrm{CdSe}(\mathrm{CdZnSe})$ QDs, формируемые в процессе самоорганизации при МВЕ-росте, использовались в качестве активной области лазерных структур, возбуждаемых оптически [2], электронным лучом [3] и при электрической накачке [4]. Показана также перспективность подобных систем для реализации излучателей неклассического света [5]. В то же время сопоставление оптических и структурных характеристик исследованных образцов вызывает ряд вопросов. К примеру, линия фотолюминесценции (PL) из массивов CdSe QDs имеет сравнительно небольшую ширину 40-60 meV при низких температуpax, характерную для узкого распределения по размерам и составу QDs. Эффективность излучения II-VI лазерных структур также достаточно высока, что могло бы реализовываться в случае массивов с низкой дисперсией параметров точек [6]. Однако проведенные исследования методом просвечивающей микроскопии (ТЕМ) свидетельствуют обратное: массивы представляют собой неоднородные ансамбли QDs, различающихся как размерами, так и содержанием Cd [7,8]. Рассмотрение таких планарных массивов в модели разупорядоченных квантовых ям (QW) проблематично, поскольку при низкой температуре рекомбинирующие носители должны быть хорошо локализованы. Согласование этих противоречивых моментов потребовало в работе [9] включения в состав QW нано-островков различных типов - изолированных, перекрывающихся и метастабильных.
Недавно нами был предложен альтернативный подход для объяснения нетривиальных свойств эпитаксиальных II-VI QDs [10,11], заключающийся в возможности ферстеровского резонансного переноса энергии (Förster resonance energy transfer, FRET) [12] из ансамбля маленьких точек, именуемых „донорами“, в ансамбль излучающих точек - „акцепторов“. В полупроводниковых системах FRET экспериментально исследовался ранее в ансамблях коллоидных QDs $[13,14]$, вертикально-связанных массивах эпитаксиальных точек [15] и QWs [16]. Теоретическое описание FRET в рамках существующих модельных представлений и необходимые ссылки могут быть найдены в работе [17]. Этот нерадиационный механизм базируется на диполь-дипольном взаимодействии между осцилляторами и не предполагает какого-либо транспорта носителей заряда. Необходимое условие для его реализации - пространственная близость доноров и акцепторов, поскольку эффективность FRET падает $\propto R^{-6}$ с расстоянием $R$. Высокая латеральная плотность QDs $\left(\sim 10^{11} \mathrm{~cm}^{-2}\right)$ способствует осуществлению FRET в массивах эпитаксиальных II-VI QDs. Отметим, что перенос энергии в структурах с II-VI QDs традиционно рассматривался как процесс туннелирования или прыжков (hopping) экситонов [18] или (при меньшей плотности) как передача энергии излучением [19].

В каноническом представлении FRET осуществляется между уровнями в ансамблях доноров и акцепторов при условии, что их спектры поглощения и излучения перекрываются [12]. Это условие выполняется в коллоидных [13] и эпитаксиальных [20] QDs. В настоящей статье мы фокусируемся на специфической разновидности FRET, возможной в неоднородных ансамблях QDs (см. рис. 1). Квантовые уровни в них выстраиваются таким образом, что существует возможность резонансного переноса энергии с основных уровней большого количества маленьких точек на возбужденные уровни 


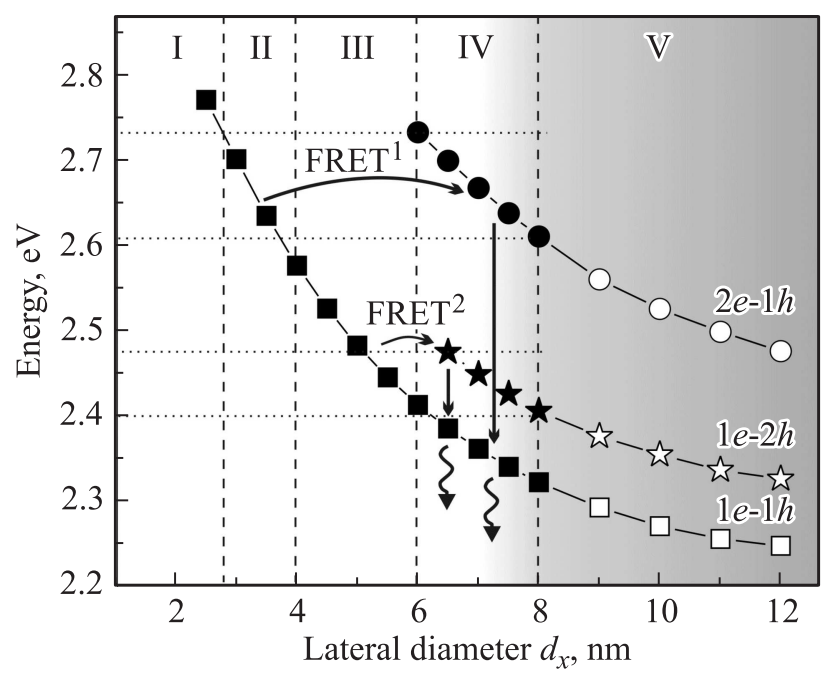

Рис. 1. Теоретические зависимости энергии основного экситонного уровня $(1 e-1 h)$ и возбужденных уровней от латерального размера $\mathrm{CdSe} / \mathrm{ZnSe}$ квантовых точек с $85 \mathrm{~mol} . \% \mathrm{Cd}$ в их центре. Области (I) $-(\mathrm{IV})$ соответствуют различной вовлеченности квантовых точек в ферстеровский резонансный перенос энергии; область (V) содержит в основном дефектные неизлучающие нано-острова (см. текст).

крупных точек с последующей быстрой релаксацией возбуждения на их основной уровень [10]. При этом число излучающих центров на единицу площади сокращается, а эффективность сбора энергии возрастает. Методами спектроскопии селективной PL, возбуждения PL (PLE) была обнаружена модификация спектров излучения при различных энергиях возбуждения, обусловленная архитектурой квантовых уровней, допускающей или не допускающей в данной спектральной области реализацию специфического типа FRET. Измерения PL с временным разрешением в структурах с двойными вставками, одна из которых функционировала как ансамбль QDs-доноров, позволили подтвердить зависимость FRET от расстояния в исследуемых системах.

\section{2. Эксперимент}

Исследовались два типа структур: 1) одиночные вставки планарных массивов QDs CdSe в $\mathrm{ZnSe}$; 2) двойные вставки, разделенные барьером толщиной $w$, изменяемой в диапазоне $6-13 \mathrm{~nm}$, что позволило изучить перенос энергии в зависимости от расстояния между QDs-донорами и QDs-акцепторами. Номинальная толщина $\mathrm{CdSe}$ в акцепторной вставке была $2.7 \mathrm{ML}$, что соответствовало концентрации $\sim 80-85 \mathrm{~mol} \% \mathrm{Cd}$ в центре квантовых точек. В донорной вставке номинальная толщина варьировалась в диапазоне $1.4-1.8 \mathrm{ML}$ ( $50 \mathrm{~mol} \% \mathrm{Cd}$ ). Данные о статистике распределения латеральных размеров QDs были получены в результате исследований методом ТЕМ-структур с одиночными вставками [21], которые показали наличие крупных нано-островов (6-8 nm и более), окруженных большим количеством маленьких точек. Вследствие сегрегации и диффузии $\mathrm{Cd}$ в процессе MBE-роста часть из них расположена выше крупных нано-островов (в пределах 5-7 nm). Предполагается, что это обстоятельство в стандартной геометрии оптических экспериментов должно облегчать возбуждение маленьких QDs с последующим переносом энергии в нижележащие нано-острова.

Структуры исследовались методом спектроскопии PLE в криостате замкнутого цикла в температурном диапазоне $20-100 \mathrm{~K}$ с использованием излучения лампы накаливания, пропущенного через монохроматор, и охлаждаемой азотом CCD-камеры. При этом непрерывно регистрировалась вся полоса возбуждаемой PL. Такие гипер-спектры PLE, помимо обычной информации о структуре пиков поглощения и вовлечении фононов в процесс излучения [10,22], позволяют проследить изменение спектра PL в зависимости от энергии возбуждения. Контрольные эксперименты по селективному возбуждению PL были выполнены при $79 \mathrm{~K}$ с использованием лазерных линий различной длины волны $(325,442,458,488,476,488 \mathrm{~nm})$ для надбарьерного $(325 \mathrm{~nm})$ и подбарьерного (остальные линии)
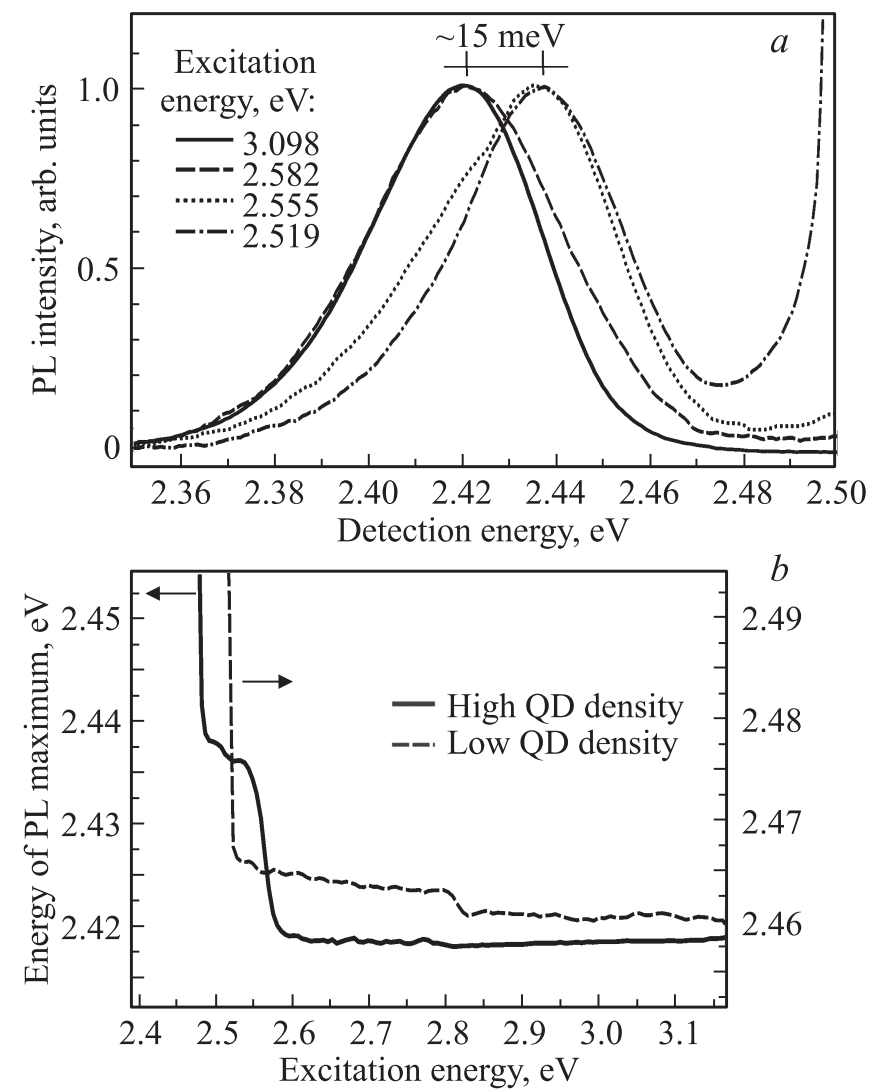

Pис. 2. (a) Спектры селективно-возбуждаемой PL для структуры с одиночной вставкой $\mathrm{CdSe}$, имеющей номинальную толщину 2.7 монослоя. (b) Энергия максимума PL в структурах с высокой и низкой плотностью QDs. Зависимости извлечены из гипер-спектров PLE. 
возбуждения. Для исключения влияния неоднородности распределения параметров QDs по структуре использовалась установка микро-PL $(\mu \mathrm{PL})$, оснащенная устройством для измерений при низкой температуре (Linkam THMS600 temperature controlled microscope stage) и объективами (Mitutoyo 50xUV, $N A=0.40$ и Mitutoyo 100xNIR, $N A=0.50)$, обеспечивающими фокусировку возбуждения в пятно диаметром $\sim 1.5 \mu \mathrm{m}$. Кинетика затухания PL исследовалась методом коррелированного счета фотонов с использованием лавинного однофотонного фотодиода PDM-100-C0E (Micro Photon Devices) и электронного TCSPC модуля SPC-130 (Becker\&Hickl). Кривые затухания PL регистрировались из максимума полосы излучения при $5 \mathrm{~K}$.

Отличительной чертой спектрального изменения селективно возбуждаемой PL, наблюдаемой как в гиперспектрах PLE, так и в спектрах $\mu \mathrm{PL}$, является смещение ее пика в коротковолновую сторону в районе $2.6 \mathrm{eV}$. Смещение достигает величины $15 \mathrm{meV}$ в одиночных вставках (рис. 2) и $23 \mathrm{meV}$ в структурах с двойными вставками (рис. 3). Подобный сдвиг линии отсутствует как в структурах с малой плотностью QDs, так и

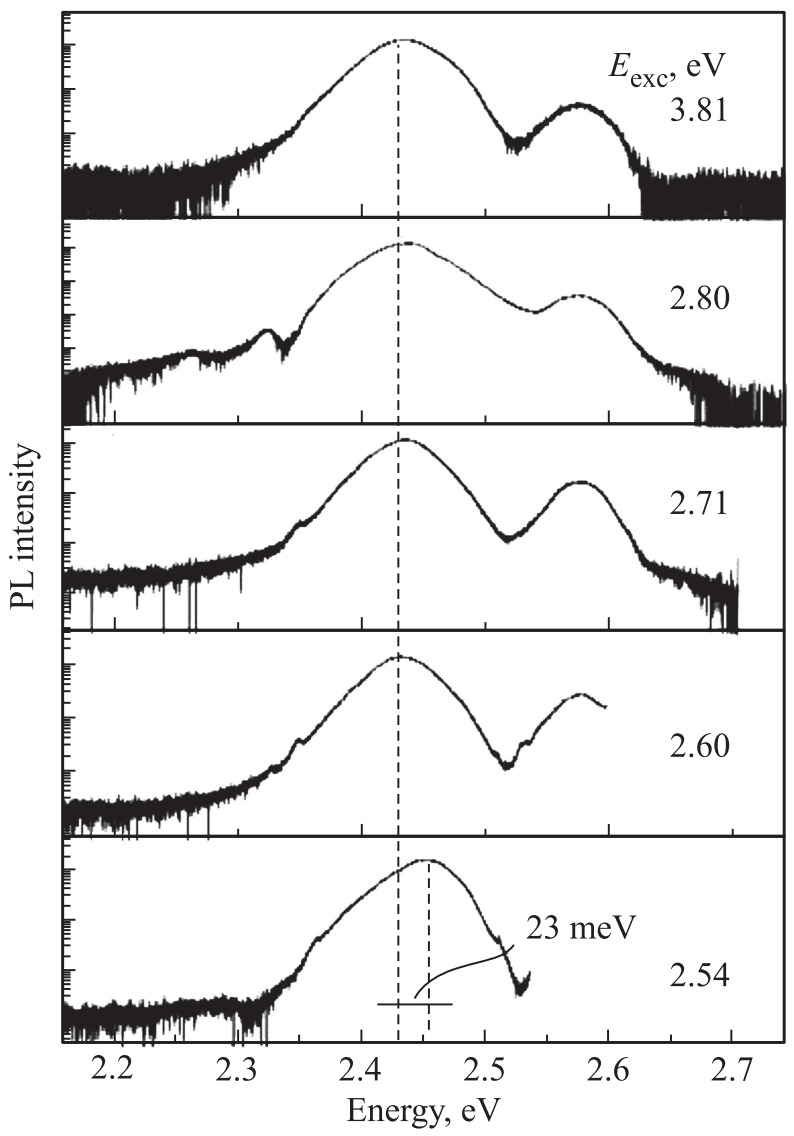

Рис. 3. Спектры $\mu$ PL-структуры с двумя вставками CdSe c номинальными толщинами 1.7 и 2.7 монослоя, измеренные при фокусировке на поверхность структуры лазерных линий с различной длиной волны. Соответствующие энергии возбуждения $E_{\text {exс }}$ приведены на графике.

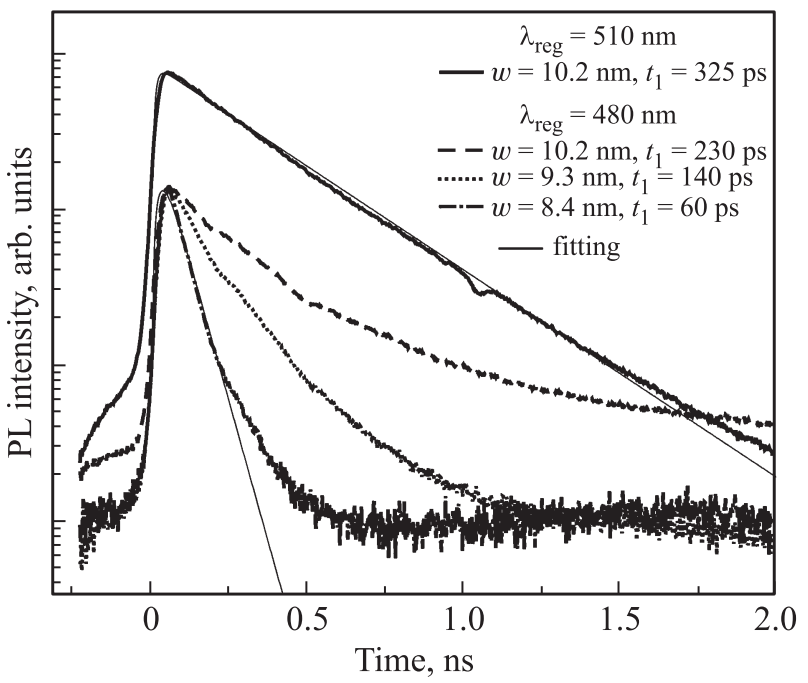

Pис. 4. Кривые затухания PL в структуре с двумя вставками, измеренные при различной ширине барьера $w$ между ними. Длины волн регистрации 480 и $510 \mathrm{~nm}$ соответствовали пику излучения из вставок толщиной 1.7 и 2.7 монослоев соответственно.

в верхнеэнергетических донорных вставках с низкой концентрацией $\mathrm{Cd}$. Как показано ниже, подобный сдвиг является индикатором существования специфического FRET, осуществляемого с участием возбужденных экситонных уровней.

Спектроскопия PL с временным разрешением структур с одиночными вставками показала наличие двух компонент в излучении - быстрой $t_{1} \sim 300$ ps и медленной $t_{2} \sim 1 \mathrm{~ns}$, соотношение между которыми зависит от энергии возбуждения и температуры. В структурах с двумя вставками времена $t_{1}$ из верхнеэнергетических вставок систематически укорачиваются при уменьшении зазора вплоть до 60 ps, что указывает на интенсификацию переноса энергии (рис. 4).

\section{3. Моделирование и анализ результатов}

Анализ результатов оптических исследований опирался на численный расчет уровней энергии электронов и дырок в сфероидных QDs с гауссовым потенциальным профилем - модели, хорошо подходящей для описания II-VI QDs [23]. Зона проводимости рассматривалась как простая с параболической изотропной дисперсией, валентная зона - в рамках сферического приближения для гамильтониана Латтинжера. Концентрация $\mathrm{Cd}$ полагалась плавно меняющейся по гауссиану от центра к краям QD. Эффективные массы электронов, тяжелых и легких дырок внутри QD считались постоянными и вычислялись согласно линейной интерполяции между $\mathrm{ZnSe}$ и $\mathrm{ZnCdSe,} \mathrm{что} \mathrm{допустимо,} \mathrm{поскольку} \mathrm{данные} \mathrm{параметры}$ близки в этих материалах. Расчет проводился с учетом 
степени анизотропии точек $\mu$, нарастающей с увеличением их латерального диаметра $d_{x}$ как $\mu=\left(d_{x}[n m]-2\right) / 10$. Размер QD в направлении роста при этом записывался как $d_{z}=d_{x}(1-2 / 3 \mu)^{0.5} /(1+4 / 3 \mu)^{0.5}$. При таком определении QDs размером $2 \mathrm{~nm}$ являются сферическими, тогда как QDs большего размера имеют форму сплюснутых в направлении роста сфероидов.

Состояния электронов и дырок (волновые функции и энергии) искались численным методом: вычислялась матрица соответствующего гамильтониана на базисе собственных функций гармонического осциллятора с жесткостью, равной жесткости потенциала квантовой точки в центре для электронов и дырок. Расчет считался сошедшимся, если небольшое изменение базиса не приводило к изменению результатов. Из найденных возможных возбужденных экситонных уровней выбирались такие, которые содержат ненулевую примесь сферической компоненты и, следовательно, допускают по правилам симметрии перенос энергии к ним от основных состояний доноров - маленьких точек.

Зависимости энергий основных и возбужденных экситонных уровней от $d_{x}$, рассчитанные для QDs с 85 mol.\% Cd в центре, приведены на рис. 1. На этих зависимостях можно выделить несколько характерных областей латеральных размеров. Затемненная область $(V)$ соответствует размерам больше критического $d_{\mathrm{cr}}$ по уровню допустимых напряжений. Эти точки изобилуют дефектами и являются, как правило, оптическими неактивными. Минимальное значение $d_{\mathrm{cr}}$ определяет нижнеэнергетическую границу излучения $\mathrm{CdSe}$ QDs при стандартной MBE технологии. Область (I) содержит QDs, для основного экситонного уровня, в которых нет соответствующего возбужденного уровня QDs-акцепторов и, следовательно, специфический механизм типа FRET $^{1}$ с быстрой релаксацией возбуждения на нижний экситонный уровень для них невозможен. Следует отметить, что граница между областью (I) и (II) спектрально приблизительно совпадает с краем подвижности (mobility edge), обсуждаемым ранее в работе [9]. Квантовые точки, размеры которых попадают в область (II), могут участвовать в переносе энергии по механизму $\mathrm{FRET}^{1}$ на возбужденные уровни $(2 e-1 h)$ в QDs в области (IV). Для данной концентрации Cd эти уровни появляются в нано-островках размером более $6 \mathrm{~nm}$. Область (III) включает QDs, которые не участвуют в процессе переноса энергии с использованием обсуждаемого механизма вплоть до появления при более низких энергиях возбужденного уровня $(1 e-2 h)$, когда становится возможным FRET $^{2}$. Тем не менее, перенос возбуждения с участием фононов [24] между основными состояниями точек разного размера в этой области возможен.

Таким образом, ниже $2.6 \mathrm{eV}$ механизм переноса энергии должен изменяться. Причем как перенос энергии с помощью фононов, так и $\mathrm{FRET}^{2}$ будут приводить к сдвигу полосы излучения в сторону высоких энергий. Первый - потому что в благоприятной для этого механизма ситуации оказываются, прежде всего, QDs на верхнеэнергетическом краю потенциальных акцепторов. Второй - поскольку возможность излучения с участием FRET $^{1}$ исчезает ниже по энергии, чем начинает действовать FRET $^{2}$ (рис. 1). Аналогичные зависимости были рассчитаны для различных концентраций $\mathrm{Cd}$ в центре точек в диапазоне 50-100 мол.\%. Их анализ позволяет говорить об общности отмеченных особенностей.

Экспериментально наблюдаемая энергия резкого сдвига максимума полосы излучения около $2.6 \mathrm{eV}$ хорошо совпадает с теоретически найденной границей смены доминирующего механизма переноса энергии. Ниже этой границы спектры PLE и $\mu \mathrm{PL}$ содержат всплески интенсивности, соответствующие переходам с участием LO-фононов. Отсутствие заметного сдвига в структурах с малой плотностью определяется резким падением эффективности FRET с увеличением расстояния между QDs. При низкой же концентрации $\mathrm{Cd}$ сдвиг не проявляется в спектрах, поскольку в этом случае возбужденные уровни появляются только в наноостровках размером более $8 \mathrm{~nm}$, попадающих в область критических размеров (V). Перенос энергии на уровни таких нано-островков (как правило, дефектных) не имеет смысла.

Ширина линии PL $\sim 50 \mathrm{meV}$, противоречащая спектрально-уширенной плотности состояний, рассчитанной в соответствии с данными ТЕМ [11], в предлагаемой модели объясняется узостью размерной области (VI), где осуществляется наиболее эффективный FRET ${ }^{1}$. Эта область, с одной стороны, ограничена критическим размером QD, необходимым для появления возбужденных уровней, с другой стороны - диаметром $d_{\mathrm{cr}}$, соответствующим началу дефектообразования в нано-островках. Поскольку число излучающих точек в области (VI), собирающих энергию со всего ансамбля, сравнительно невелико, оказывается возможным наблюдение ограниченного числа узких линий вместо сплошной полосы излучения, ожидаемой при высокой латеральной плотности QDs [10].

\section{4. Заключение}

Полученные результаты и проведенный анализ проясняют ряд противоречивых оптических свойств эпитаксиальных II-VI QDs. Показано, что существенное влияние на кинетику PL в плотных массивах $\mathrm{CdSe} / \mathrm{ZnSe}$ QDs оказывает ферстеровский резонансный перенос возбуждения. Установлено, что резкий сдвиг максимума полосы излучения связан с изменением доминирующего механизма переноса энергии в неоднородных ансамблях точек. Можно предположить, что направленное создание массивов QDs с бимодальным распределением размеров будет полезно для спектрально-селективного увеличения эффективности нано-лазеров и других нанофотонных приборов. 


\section{Список литературы}

[1] S.V. Ivanov, A.A. Toropov, T.V. Shubina, S.V. Sorokin, A.V. Lebedev, I.V. Sedova, P.S. Kop'ev. J. Appl. Phys. 83, 3168 (1998).

[2] S.V. Ivanov, A.A. Toropov, S.V. Sorokin, T.V. Shubina, I.V. Sedova, A.A. Sitnikova, P.S. Kop'ev, Z.I. Alferov, A. Waag, H.-J. Lugauer, G. Reuscher, M. Keim, G. Landwehr. Appl. Phys. Lett. 74, 498 (1999).

[3] S.V. Sorokin, S.V. Gronin, I.V. Sedova, M.V. Rakhlin, M.V. Baidakova, P.S. Kop'ev, A.G. Vainilovich, E.V. Lutsenko, G.P. Yablonskii, N.A. Gamov, E.V. Zhdanova, M.M. Zverev, S.S. Ruvimov, S.V. Ivanov. Semiconductors 49, 331 (2015).

[4] M. Klude, T. Passow, R. Kröger, D. Hommel. Electron. Lett. 37, 1119 (2001).

[5] O. Fedorych, C. Kruse, A. Ruban, D. Hommel, G. Bacher, T. Kümmell. Appl. Phys. Lett. 100, 061114 (2012).

[6] L.V. Asryan, R.A. Suris. Semiconductors 38, 1 (2004).

[7] N. Peranio, A. Rosenauer, D. Gerthsen, S.V. Sorokin, I.V. Sedova, S.V. Ivanov. Phys. Rev. B 61, 16015 (2000).

[8] D. Litvinov, A. Rosenauer, D. Gerthsen, N.N. Ledentsov. Phys. Rev. B 61, 16819 (2000).

[9] A. Klochikhin, A. Reznitsky, B. Dal Don, H. Priller, H. Kalt, C. Klingshirn, S. Permogorov, S. Ivanov. Phys. Rev. B 69, 085308 (2004).

[10] T.V. Shubina, A.V. Rodina, M.A. Semina, A.A. Golovatenko, A.A. Toropov, M.V. Rakhlin, I.V. Sedova, S.V. Sorokin, S.V. Gronin, A.A. Sitnikova, D.I. Kuritsyn, S.M. Sergeev, Z.F. Krasil'nik, S.V. Ivanov. Phys. Status Solidi B, (2016) / DOI 10.1002/pssb.201600095.

[11] A.A. Golovatenko, M.A. Semina, A.V. Rodina, T.V. Shubina. Acta Phys. Polon. A 129, 107 (2016).

[12] T. Förster. Ann. Physik 437, 55 (1948).

[13] S.A. Crooker, J.A. Hollingsworth, S. Tretiak, V.I. Klimov. Phys. Rev. Lett. 89, 186802 (2002).

[14] F. Liu, A.V. Rodina, D.R. Yakovlev, A.A. Golovatenko, A. Greilich, E.D. Vakhtin, A. Susha, A.L. Rogach, Y.G. Kusrayev, M. Bayer. Phys. Rev. B 92, 125403 (2015).

[15] T.S. Shamirzaev, D.S. Abramkin, D.V. Dmitriev, A.K. Gutakovskii. Appl. Phys. Lett. 97, 263102 (2010).

[16] J.J. Rindermann, G. Pozina, B. Monemar, L. Hultman, H. Amano, P.G. Lagoudakis. Phys. Rev. Lett. 107, 236805 (2011).

[17] A.N. Poddubny, A.V. Rodina. ЖЭТФ 149, 614 (2016).

[18] K.P. Korona, P. Wojnar, J.A. Gaj, G. Karczewski, J. Kossut, J. Kuhl. Solid State Commun. 133, 369 (2005).

[19] M. Scheibner, T. Schmidt, L. Worschech, A. Forchel, G. Bacher, T. Passow, D. Hommel. Nature Phys. 3, 106 (2007).

[20] T. Kazimierczuk, J. Suffczyński, A. Golnik, J.A. Gaj, P. Kossacki. Phys. Rev. B 79, 153301 (2009).

[21] T.V. Shubina, A.V. Rodina, M.A. Semina, A. A. Golovatenko, A.A. Toropov, M.V. Rakhlin I.V. Sedova, S.V. Sorokin, A.A. Sitnikova, D.A. Kirilenko, V.Kh. Kaibyshev, P.S. Kop’ev, S.V. Ivanov. Proc. 23 Int. Symp. „Nanostructures: Physics and Technology“ Saint-Petersburg (2015). C. 86.

[22] A. Reznitsky, M. Eremenko, I.V. Sedova, S.V. Sorokin, S.V. Ivanov. Phys. Status Solidi B 252, 1717 (2015).

[23] M.A. Semina, A.A. Golovatenko, A.V. Rodina. Phys. Rev. B 93, 045409 (2016)

[24] P. Hernandez-Martinez, A. Govorov, H. Demir. J. Phys. Chem. C 117, 10203 (2013). 